\title{
Sums of coefficients of Hecke series
}

\author{
by \\ Aleksandar Ivić (Belgrade) and Tom Meurman (Turku)
}

1. Introduction and statement of results. Let

$$
H_{j}(s)=\sum_{n=1}^{\infty} t_{j}(n) n^{-s} \quad(\operatorname{Re} s>1)
$$

be the Hecke series attached to the Maass wave form $\varphi_{j}(z)$ corresponding to the eigenvalue $\lambda_{j}=\kappa_{j}^{2}+1 / 4\left(\kappa_{j}>0, j \geq 1\right)$ of the non-Euclidean Laplacian acting on the space of all non-holomorphic automorphic functions with respect to $\operatorname{SL}(2, \mathbb{Z})$. The set $\left\{\varphi_{j}\right\}$ forms an orthonormal base of the subspace spanned by all cusp forms. The $t_{j}(n)$ 's are real-valued and are the eigenvalues of the Hecke operator $T(n)$, which means that, for $\operatorname{Im} z>0$,

$$
\left(T(n) \varphi_{j}\right)(z)=n^{-1 / 2} \sum_{a d=n} \sum_{b=1}^{d} \varphi_{j}\left(\frac{a z+b}{d}\right)=t_{j}(n) \varphi_{j}(z) .
$$

The series in (1.1) converges absolutely only for $\operatorname{Re} s>1$, but the function $H_{j}(s)$ can be continued analytically to an entire function over the $s$-plane. It satisfies the functional equation

$$
H_{j}(s)=X_{j}(s) H_{j}(1-s)
$$

with

(1.3) $X_{j}(s)=2^{2 s-1} \pi^{2 s-2} \Gamma\left(1-s+i \kappa_{j}\right) \Gamma\left(1-s-i \kappa_{j}\right)\left(\varepsilon_{j} \operatorname{ch}\left(\pi \kappa_{j}\right)-\cos (\pi s)\right)$.

Here $\varepsilon_{j}$ is the parity sign of the Maass wave form $\varphi_{j}$, namely

$$
\varepsilon_{j}= \begin{cases}1 & \text { if } \varphi_{j}(-x+i y)=\varphi_{j}(x+i y), \\ -1 & \text { if } \varphi_{j}(-x+i y)=-\varphi_{j}(x+i y) .\end{cases}
$$

Starting with the pioneering works of N. V. Kuznetsov [17]-[19] and H. Iwaniec [11], spectral theory and Hecke series have become increasingly important in analytic number theory. Recently Y. Motohashi made heavy use of this theory (see [23], [24], [10] and Ch. 5 of [9]) to establish precise

Research of the first author financed by the Mathematical Institute of Belgrade. 
formulas for the fourth moment of $|\zeta(1 / 2+i t)|$. A comprehensive account on spectral theory and Maass wave forms is found in the works of H. Iwaniec [12], [13].

The aim of this work is to study the partial sum of the coefficients in (1.1), namely

$$
T_{j}(x)=\sum_{n \leq x}^{\prime} t_{j}(n),
$$

its mean square integral, and the summatory function of $t_{j}^{2}(n)$. In (1.4) the dash denotes that the last term in the sum is to be halved if $x$ is an integer. The accent in our investigations is on the fact, often encountered in applications, that $\kappa_{j}$ does not have to be fixed, but may vary with $x$. Naturally this causes difficulties, since all estimates must then be uniform in $\kappa_{j}$. The results that we obtain (see Theorems 1-3 below) are the sharpest ones hitherto.

If $\kappa_{j}$ is fixed, then it was shown by Hafner-Ivić [7] that

$$
T_{j}(x) \ll x^{2 / 5} .
$$

Such a result follows from a general theorem of Chandrasekharan-Narasimhan [3] on Dirichlet series with a functional equation containing gammafactors. The second author proved (Corollary to Theorem 1 in [20]) that

$$
T_{j}(x)-H_{j}(0) \ll_{\varepsilon} x^{(1+\alpha) / 3+\varepsilon} \quad\left(\kappa_{j} \leq x^{1 / 2}\right),
$$

where as usual $\varepsilon$ will denote arbitrarily small, positive constants, not necessarily the same at each occurrence. In fact, $[20]$ treats the more general case when $t_{j}(n)$ in (1.4) is multiplied by an exponential factor $\exp (2 \pi i h n / k)$. In (1.6), $\alpha \geq 0$ is a constant for which

$$
t_{j}(n) \ll_{\varepsilon} n^{\alpha+\varepsilon}
$$

uniformly in $\kappa_{j}$. The famous Ramanujan-Petersson conjecture for Maass wave forms asserts that $\alpha=0$. It is known that $\alpha \leq 1 / 5$ from the work of J.-P. Serre (see [22] and [25]), and recently D. Bump et al. [2] proved that $\alpha \leq 5 / 28$. In bounding $T_{j}(x)$ it will be enough to consider the range $x^{1 / 2}<\kappa_{j} \leq x$. Namely, Deshouillers-Iwaniec [5] proved that

$$
\sum_{n \leq x}\left|\varrho_{j}(n)\right|^{2} \ll \operatorname{ch}\left(\pi \kappa_{j}\right)\left(x+\kappa_{j}\right) \log \left(2+x / \kappa_{j}\right)
$$

uniformly in $\kappa_{j}$, where $\varrho_{j}(n)$ denotes the $n$th Fourier coefficient of the Maass wave form $\varphi_{j}(z)$. Also for the quantity

$$
\alpha_{j}=\left|\varrho_{j}(1)\right|^{2} / \operatorname{ch}\left(\pi \kappa_{j}\right)
$$


H. Iwaniec [14] proved the lower bound

$$
\alpha_{j} \gg_{\varepsilon} \kappa_{j}^{-\varepsilon} \text {. }
$$

Now if one uses

$$
\varrho_{j}(n)=\varrho_{j}(1) t_{j}(n)
$$

and the multiplicative property

$$
t_{j}(m) t_{j}(n)=\sum_{d \mid(m, n)} t_{j}\left(m n / d^{2}\right) \quad(m \geq 1, n \geq 1),
$$

then it follows from (1.8) and (1.10) (see H. Iwaniec [15]) that, uniformly in $\kappa_{j}$,

$$
\sum_{n \leq x} t_{j}^{2}(n) \ll_{\varepsilon} \kappa_{j}^{\varepsilon} x
$$

Hence from (1.13) one obtains, by using the Cauchy-Schwarz inequality, that uniformly in $\kappa_{j}$,

$$
\sum_{n \leq x} t_{j}(n) \ll_{\varepsilon} \kappa_{j}^{\varepsilon} x
$$

Our first result improves (1.14) for $\kappa_{j} \leq x$ :

Theorem 1. For any given $\varepsilon>0$ and $x^{1 / 2}<\kappa_{j} \leq x$ we have

$$
T_{j}(x)=\sum_{n \leq x}^{\prime} t_{j}(n) \ll_{\varepsilon} \kappa_{j}^{1+\varepsilon} .
$$

This is compatible with (1.6), since by the functional equation (1.2) we have $\left|H_{j}(0)\right|=\left|X_{j}(0) H_{j}(1)\right|$, and by (1.3),

$$
\left|X_{j}(0)\right|=\frac{\kappa_{j}}{2 \pi}\left|\frac{\varepsilon_{j} \operatorname{ch}\left(\pi \kappa_{j}\right)-1}{\operatorname{sh}\left(\pi \kappa_{j}\right)}\right| \asymp \kappa_{j},
$$

while on using (1.6) and (1.14) one finds that

$$
\begin{aligned}
H_{j}(1) & =\sum_{n=1}^{\infty} t_{j}(n) n^{-1}=\int_{1-0}^{\infty} u^{-2}\left(\sum_{n \leq u} t_{j}(n)\right) d u=\int_{1-0}^{\kappa_{j}^{4}}+\int_{\kappa_{j}^{4}}^{\infty} \\
& =O_{\varepsilon}\left(\kappa_{j}^{\varepsilon}\right)+H_{j}(0) \kappa_{j}^{-2}=O_{\varepsilon}\left(\kappa_{j}^{\varepsilon}\right)+X_{j}(0) H_{j}(1) \kappa_{j}^{-2} .
\end{aligned}
$$

Thus $H_{j}(1) \ll_{\varepsilon} \kappa_{j}^{\varepsilon}$ and consequently $\kappa_{j}\left|H_{j}(1)\right| \ll\left|H_{j}(0)\right| \ll_{\varepsilon} \kappa_{j}^{1+\varepsilon}$. This shows that $H_{j}(0)$ may be large if $\kappa_{j}$ is not fixed. Therefore, apart from $\varepsilon$, the bound in (1.15) is in a certain sense the best one can hope for.

Our mean square result on $T_{j}(x)$ is contained in 
Theorem 2. If $X<Y \leq 2 X, \kappa_{j} \leq X^{1 / 2}$ and $h\left(\kappa_{j}\right)\left(\ll \kappa_{j}^{\varepsilon-1 / 2}\right)$ is given by (4.6), then for any given $\varepsilon>0$ we have, uniformly in $\kappa_{j}$,

$$
\begin{aligned}
& \int_{X}^{Y}\left(T_{j}(x)-H_{j}(0)-h\left(\kappa_{j}\right)\right)^{2} d x \\
& =\frac{1}{4 \pi^{2}} \sum_{n=1}^{\infty} t_{j}^{2}(n) \int_{X}^{Y} x^{1 / 2}\left(n+\frac{1}{x}\left(\frac{\kappa_{j}}{2 \pi}\right)^{2}\right)^{-3 / 2} d x+O_{\varepsilon}\left(X^{1+\varepsilon}\right) .
\end{aligned}
$$

The analogue of Theorem 2 holds for holomorphic cusp forms. This is due to M. Jutila [16] (see Th. 1.2 and Notes to Ch. 1).

From (1.16) we obtain without difficulty the following

COROllary 1. For $\kappa_{j} \leq X^{1 / 2}$ and any given $\varepsilon>0$ we have, uniformly in $\kappa_{j}$,

$$
\begin{aligned}
\int_{X}^{2 X} T_{j}^{2}(x) d x=\left\{\frac{\sqrt{8}-1}{6 \pi^{2}} \sum_{n=1}^{\infty} t_{j}^{2}(n) n^{-3 / 2}\right\} X^{3 / 2}+H_{j}^{2}(0) X \\
+O_{\varepsilon}\left\{\left(\kappa_{j}^{2} X^{1 / 2}+\kappa_{j}^{1 / 2} X+\kappa_{j} X^{3 / 4}\right) X^{\varepsilon}\right\} .
\end{aligned}
$$

If $\kappa_{j}$ is fixed, then

$$
\int_{1}^{X} T_{j}^{2}(x) d x=\left\{\frac{1}{6 \pi^{2}} \sum_{n=1}^{\infty} t_{j}^{2}(n) n^{-3 / 2}\right\} X^{3 / 2}+O_{\varepsilon}\left(X^{1+\varepsilon}\right) .
$$

Note that, for $Y=2 X$, the main term on the right-hand side of (1.16) is

$$
\geq \frac{1}{4 \pi^{2}} t_{j}^{2}(1) \int_{X}^{2 X} x^{1 / 2} d x=\frac{\sqrt{8}-1}{6 \pi^{2}} X^{3 / 2} .
$$

Suppose now that $T_{j}(x)=o\left(x^{1 / 4}\right)$ as $x \rightarrow \infty$. Since $H_{j}(0) \ll_{\varepsilon} \kappa_{j}^{1+\varepsilon}$, for $\kappa_{j} \leq x^{1 / 4-\varepsilon_{1}}$ and any given $\varepsilon_{1}>0$ we deduce that the left-hand side of $(1.16)$ is $o\left(X^{3 / 2}\right)$, which is a contradiction. Thus we obtain

COROLlary 2. If $\kappa_{j} \leq x^{1 / 4-\varepsilon_{1}}$ for any given $\varepsilon_{1}>0$, then $T_{j}(x)=$ $\Omega\left(x^{1 / 4}\right)$.

To assess the strength of Theorem 2 it may be remarked that (1.18) is analogous to the mean square formula

$$
\int_{1}^{X} \Delta^{2}(x) d x=\left\{\frac{1}{6 \pi^{2}} \sum_{n=1}^{\infty} d^{2}(n) n^{-3 / 2}\right\} X^{3 / 2}+O\left(X \log ^{4} X\right),
$$

where

$$
\Delta(x)=\sum_{n \leq x}^{\prime} d(n)-x(\log x+2 \gamma-1)-1 / 4
$$


is the error term in the classical divisor problem. Here $d(n)$ is the number of divisors of $n$, and $\gamma$ is Euler's constant. The omega-result $T_{j}(x)=\Omega\left(x^{1 / 4}\right)$ is the analogue of the classical omega-result $\Delta(x)=\Omega\left(x^{1 / 4}\right)$, which follows from (1.19). For $\Delta(x)$ even sharper omega-results are known, but their analogues for $T_{j}(x)$ are hard to obtain, since little is known about the arithmetical structure of the $t_{j}(n)$ 's.

The error term in (1.19) is the sharpest one known. It is due to E. Preissmann [26], while K.-C. Tong [30] and T. Meurman [21] had $O\left(X \log ^{5} X\right)$. The method of [21] was a substantial simplification of Tong's work, and yielded also the analogue of (1.19) for the important function

$$
E(T):=\int_{0}^{T}|\zeta(1 / 2+i t)|^{2} d t-T(\log (T /(2 \pi))+2 \gamma-1) .
$$

It can clearly be used for general mean square formulas, for example the ones considered by Chandrasekharan-Narasimhan [4]. A variant of this method will be used for the proof of Theorem 2. Since the function $\Delta(x)$ is less difficult to handle than $T_{j}(x)$, the error term in (1.16) appears to be the limit of the present methods.

To deal with the summatory function of $t_{j}^{2}(n)$ we introduce the arithmetical function

$$
c_{j}(n):=\sum_{d^{2} \mid n}\left|\varrho_{j}\left(n / d^{2}\right)\right|^{2} .
$$

In terms of the generating Dirichlet series, (1.20) is equivalent to

$$
C_{j}(s)=\zeta(2 s) L_{j}(s)
$$

where

$$
L_{j}(s):=\sum_{n=1}^{\infty}\left|\varrho_{j}(n)\right|^{2} n^{-s}, \quad C_{j}(s):=\sum_{n=1}^{\infty} c_{j}(n) n^{-s} .
$$

From (1.11) and (1.13) we obtain, uniformly in $\kappa_{j}$,

$$
\sum_{n \leq x}\left|\varrho_{j}(n)\right|^{2} \ll_{\varepsilon} \alpha_{j} \kappa_{j}^{\varepsilon} \operatorname{ch}\left(\pi \kappa_{j}\right) x, \quad \sum_{n \leq x} c_{j}(n) \ll_{\varepsilon} \alpha_{j} \kappa_{j}^{\varepsilon} \operatorname{ch}\left(\pi \kappa_{j}\right) x,
$$

so that both series in (1.22) converge absolutely for $\operatorname{Re} s>1$. In fact, $C_{j}(s)$ possesses meromorphic continuation having only a simple pole at $s=1$ with residue $2 \operatorname{ch}\left(\pi \kappa_{j}\right)$. It satisfies the functional equation (see N. V. Proskurin [27] for a proof of these facts)

$$
\pi^{-2 s} C_{j}(s) \theta_{j}(s)=\pi^{-2(1-s)} C_{j}(1-s) \theta_{j}(1-s),
$$

where

$$
\theta_{j}(s)=\Gamma\left(s / 2+i \kappa_{j}\right) \Gamma\left(s / 2-i \kappa_{j}\right) \Gamma^{2}(s / 2) .
$$


The proof of (1.24)-(1.25) is obtained by the so-called Rankin-Selberg convolution method (see D. Bump [1] for an extensive account).

Suppose now that $\kappa_{j}$ is fixed. As in the proof of (1.5) we can appeal to the method of Chandrasekharan-Narasimhan [3] to evaluate the summatory function of $c_{j}(n)$. In their notation $q=r=\delta=1, A=2, u=3 / 8$. We use their Remark (5.5), since (e.g. by the Wiener-Ikehara-Tauberian theorem) $\sum_{n \leq x} c_{j}(n)=(1+o(1)) 2 x \operatorname{ch}\left(\pi \kappa_{j}\right)$ as $x \rightarrow \infty$, so that $a=b=1$. Thus (4.2) of [3] yields

$$
\begin{aligned}
\sum_{n \leq x} c_{j}(n) & =2 x \operatorname{ch}\left(\pi \kappa_{j}\right)+O\left(x^{3 / 8+3 \eta / 2}\right)+O\left(x^{3 / 4-\eta}\right) \\
& =2 x \operatorname{ch}\left(\pi \kappa_{j}\right)+O\left(x^{3 / 5}\right)
\end{aligned}
$$

if $\eta=3 / 20$. In view of (1.9), (1.11) and (1.20) it is seen that from (1.26) one obtains by a convolution argument

$$
\sum_{n \leq x} t_{j}^{2}(n)=\frac{12 x}{\pi^{2} \alpha_{j}}+O\left(x^{3 / 5}\right)
$$

This result corresponds to R. A. Rankin's classical theorem [28] for holomorphic cusp forms. H. Iwaniec [12], [13] states (1.27) with the incorrect constant $6 /\left(\pi^{2} \alpha_{j}\right)$ in the main term, as is also done on p. 244 of [9].

When $\kappa_{j}$ is not fixed, we naturally cannot expect to obtain formulas for the above sum with error term as good as $O\left(x^{3 / 5}\right)$. What we shall prove is

Theorem 3. If $\alpha$ is the constant defined by (1.7) and $\kappa_{j} \leq x^{1-\alpha}$ then for any given $\varepsilon>0$ we have, uniformly in $\kappa_{j}$,

$$
\begin{aligned}
\sum_{n \leq x} t_{j}^{2}(n)= & \frac{12 x}{\pi^{2} \alpha_{j}}+O_{\varepsilon}\left(x ^ { \varepsilon } \left(\kappa_{j}^{1 /(2-2 \alpha)} x^{1 / 2}\right.\right. \\
& \left.\left.+\min \left(\kappa_{j}^{(1+10 \alpha) /(3+6 \alpha)} x^{1 / 2}+x^{(3+6 \alpha) / 5}, \kappa_{j}^{-1} x^{1+2 \alpha}\right)\right)\right) .
\end{aligned}
$$

The asymptotic formula (1.28) shows that, under the truth of the Ramanujan-Petersson conjecture $\alpha=0$, we can extend (1.27) (with an additional $\varepsilon$-factor) to the range $\kappa_{j} \leq x^{1 / 5}$, namely

$$
\sum_{n \leq x} t_{j}^{2}(n)=\frac{12 x}{\pi^{2} \alpha_{j}}+O_{\varepsilon}\left(x^{3 / 5+\varepsilon}\right) \quad\left(\kappa_{j} \leq x^{1 / 5}\right) .
$$

If $1 / 10<\alpha<1 / 2$ and $\kappa_{j} \leq x^{(3+6 \alpha) / 8}$ then the error terms in (1.28) are

$$
O_{\varepsilon}\left(x^{\varepsilon}\left(\kappa_{j}^{(1+10 \alpha) /(3+6 \alpha)} x^{1 / 2}+x^{(3+6 \alpha) / 5}\right)\right) .
$$

Note that (1.28) improves the result of N. V. Proskurin [27] (in the special case when $\kappa_{j_{1}}=\kappa_{j_{2}}=\kappa_{j}$ ), who investigated the summatory function of $\varrho_{j_{1}}(n) \overline{\varrho_{j_{2}}(n)}$. 
2. Proof of Theorem 1. For the proof of Theorem 1 we shall need the following

Lemma 1. Let $s=\sigma+i t,-\varepsilon \leq \sigma \leq 1+\varepsilon$ for a small, fixed $\varepsilon>0$, and $t \geq 1$. Then

$$
H_{j}(s) \ll_{\varepsilon}\left(\kappa_{j}+t\right)^{1-\sigma+2 \varepsilon},
$$

and if $\left|t-\kappa_{j}\right| \geq 2$, then

$$
\begin{aligned}
X_{j}(s) & =\frac{H_{j}(s)}{H_{j}(1-s)} \\
& =\pi^{2 s-1} c_{t}\left(\frac{1}{4}\left|t^{2}-\kappa_{j}^{2}\right|\right)^{1 / 2-s}\left|\frac{t-\kappa_{j}}{t+\kappa_{j}}\right|^{i \kappa_{j}} e^{2 i t}\left(1+O\left(\frac{1}{\left|t-\kappa_{j}\right|}\right)\right),
\end{aligned}
$$

where

$$
c_{t}= \begin{cases}\varepsilon_{j} & \text { if } t<\kappa_{j}-2, \\ i & \text { if } t>\kappa_{j}+2 .\end{cases}
$$

Proof. By using standard properties of the gamma-function one can rewrite the functional equation (1.2) as

$$
\begin{aligned}
\theta_{j}(s) H_{j}(s) & =\theta_{j}(1-s) H_{j}(1-s) & & \text { if } \varepsilon_{j}=1, \\
\theta_{j}(1+s) H_{j}(s) & =-\theta_{j}(2-s) H_{j}(1-s) & & \text { if } \varepsilon_{j}=-1,
\end{aligned}
$$

where

$$
\theta_{j}(s)=\pi^{-s} \Gamma\left(\frac{s}{2}+\frac{i}{2} \kappa_{j}\right) \Gamma\left(\frac{s}{2}-\frac{i}{2} \kappa_{j}\right) .
$$

Hence by using Stirling's formula in the form

$$
\begin{array}{r}
\Gamma(s)=\sqrt{2 \pi}|t|^{\sigma-1 / 2} \exp \left\{-\frac{\pi}{2}|t|+i\left(t \log |t|-t+\frac{\pi t}{2|t|}\left(\sigma-\frac{1}{2}\right)\right)\right\} \\
\times\left(1+O\left(\frac{1}{|t|}\right)\right) \quad(|t| \geq 1),
\end{array}
$$

one obtains (2.2). By (1.13) we have

$$
\left|H_{j}(1+\varepsilon+i t)\right| \leq \sum_{n=1}^{\infty}\left|t_{j}(n)\right| n^{-1-\varepsilon} \ll_{\varepsilon} \kappa_{j}^{\varepsilon},
$$

and by $(2.2)$,

$$
\left|H_{j}(-\varepsilon+i t)\right|=\left|H_{j}(1+\varepsilon+i t) X_{j}(-\varepsilon+i t)\right| \ll_{\varepsilon} \kappa_{j}^{\varepsilon}\left(\kappa_{j}+t\right)^{1+2 \varepsilon} .
$$

Therefore (2.1) follows by the Phragmén-Lindelöf principle (see (A.36) of $[8])$.

We now pass to the proof of Theorem 1 , supposing that $x^{1 / 2}<\kappa_{j} \leq x$ and starting from the Perron inversion formula for Dirichlet series (see e.g. 
(A.10) of [8]). If $x^{\varepsilon_{1}} \leq T \leq x^{1-\varepsilon_{1}}$ and $\alpha$ is the constant defined by (1.7), then by the residue theorem we have

$$
\begin{aligned}
\sum_{n \leq x} t_{j}(n) & \frac{1}{2 \pi i} \int_{1+\varepsilon-i T}^{1+\varepsilon+i T} H_{j}(s) x^{s} \frac{d s}{s}+O_{\varepsilon}\left(x^{1+\alpha+\varepsilon} T^{-1}\right) \\
= & \frac{1}{2 \pi i} \int_{-\varepsilon-i T}^{-\varepsilon+i T} H_{j}(s) x^{s} \frac{d s}{s}+H_{j}(0) \\
& +O_{\varepsilon}\left(x^{1+\alpha+\varepsilon} T^{-1}+\max _{-\varepsilon \leq \sigma \leq 1+\varepsilon}\left(\kappa_{j}+T\right)^{1-\sigma+2 \varepsilon} x^{\sigma} T^{-1}\right) \\
= & \frac{1}{2 \pi i} \int_{-\varepsilon-i T}^{-\varepsilon+i T} H_{j}(1-s) X_{j}(s) x^{s} \frac{d s}{s} \\
& +O_{\varepsilon}\left(\kappa_{j}^{1+\varepsilon}+x^{1+\alpha+\varepsilon} T^{-1}\right) \\
= & \sum_{n \leq \kappa_{j}^{2} / x} t_{j}(n)\left(\frac{1}{2 \pi i} \int_{-\varepsilon-i T}^{-\varepsilon+i T} n^{s-1} X_{j}(s) x^{s} \frac{d s}{s}\right) \\
& +\sum_{n>\kappa_{j}^{2} / x} t_{j}(n) n^{-\varepsilon-1} \operatorname{Re}\left\{\frac{1}{\pi x^{\varepsilon}} \int_{1}^{T}(x n)^{i t} X_{j}(-\varepsilon+i t) \frac{d t}{-\varepsilon+i t}\right\} \\
& +O_{\varepsilon}\left(\kappa_{j}^{1+\varepsilon}+x^{1+\alpha+\varepsilon} T^{-1}\right) \\
= & \sum_{1}+\sum_{2}+O_{\varepsilon}\left(\kappa_{j}^{1+\varepsilon}+x^{1+\alpha+\varepsilon} T^{-1}\right),
\end{aligned}
$$

say. Here we used (2.1), the functional equation (1.2), and exchanged the order of summation and integration in view of the absolute convergence of $H_{j}(1-s)$ for $\operatorname{Re} s<0$. In the integrals in $\sum_{1}$, which is a non-empty sum since $\kappa_{j}>x^{1 / 2}$, we replace the segment of integration $[-\varepsilon-i T,-\varepsilon+i T]$ by the segment $[1 / 2-i T, 1 / 2+i T]$. By the residue theorem, (1.3) and (2.2) we obtain

$$
\begin{aligned}
\frac{1}{2 \pi i} \int_{-\varepsilon-i T}^{-\varepsilon+i T} & n^{s} X_{j}(s) x^{s} \frac{d s}{s} \\
& =-X_{j}(0)+O\left\{(n x)^{1 / 2} \log T+\max _{-\varepsilon \leq \sigma \leq 1 / 2}(n x)^{\sigma} T^{-1}\left(\kappa_{j}+T\right)^{1-2 \sigma}\right\} \\
& \ll \kappa_{j}+(n x)^{1 / 2} \log T .
\end{aligned}
$$


Therefore by using (1.13) we obtain

$$
\begin{aligned}
\sum_{1} & \ll \kappa_{j} \sum_{n \leq \kappa_{j}^{2} / x}\left|t_{j}(n)\right| n^{-1}+x^{1 / 2} \log T \sum_{n \leq \kappa_{j}^{2} / x}\left|t_{j}(n)\right| n^{-1 / 2} \\
& \ll_{\varepsilon} \kappa_{j}^{1+2 \varepsilon} .
\end{aligned}
$$

To estimate $\sum_{2}$ we use (2.2) to write

$$
\begin{aligned}
\int_{1}^{T}(x n)^{i t} X_{j}(-\varepsilon+i t) & \frac{d t}{-\varepsilon+i t} \\
= & \int_{1}^{T}(x n)^{i t} c_{t} \pi^{-1-2 \varepsilon+2 i t}\left(\frac{1}{4}\left|t^{2}-\kappa_{j}^{2}\right|\right)^{1 / 2+\varepsilon-i t}\left|\frac{t-\kappa_{j}}{t+\kappa_{j}}\right|^{i \kappa_{j}} e^{2 i t} \frac{d t}{i t} \\
& +O_{\varepsilon}\left(\kappa_{j}^{1+2 \varepsilon}\right) \\
= & \int_{1}^{T} d_{t}\left|t^{2}-\kappa_{j}^{2}\right|^{1 / 2+\varepsilon} e^{i F(t)} \frac{d t}{t}+O_{\varepsilon}\left(\kappa_{j}^{1+2 \varepsilon}\right),
\end{aligned}
$$

where $d_{t}$ is piecewise constant,

$$
F(t)=F\left(t ; \kappa_{j}, x, n\right):=2 t+\kappa_{j} \log \left|\frac{t-\kappa_{j}}{t+\kappa_{j}}\right|-t \log \left|t^{2}-\kappa_{j}^{2}\right|+t \log \left(4 \pi^{2} x n\right),
$$

and $*$ indicates that the interval $\left[\kappa_{j}-2, \kappa_{j}+2\right]$ is excluded from integration if it intersects $[1, T]$ (its contribution is trivially $\ll 1$ ). The total error coming from the error term in (2.2) and from replacing $-\varepsilon+i t$ by $i t$ is $\ll_{\varepsilon} \kappa_{j}^{1+2 \varepsilon}$. We divide the interval of integration in the last integral in $O(\log T)$ subintervals of the form $\left[T_{1}, T_{2}\right]$, where $1 \ll T_{1}<T_{2} \leq 2 T_{1} \leq T$, and note that

$$
F^{\prime}(t)=\log \frac{4 \pi^{2} x n}{\left|t^{2}-\kappa_{j}^{2}\right|}, \quad F^{\prime \prime}(t)=\frac{-2 t}{t^{2}-\kappa_{j}^{2}} .
$$

In the subinterval of $\left[T_{1}, T_{2}\right]$ in which

$$
\left|F^{\prime \prime}(t)\right| \geq 2 / t
$$

we estimate the corresponding integral by the second derivative test (Lemma 2.2 of [8]) as

$$
\ll_{\varepsilon}\left(T_{1}+\kappa_{j}\right)^{1+2 \varepsilon} T_{1}^{-1 / 2} \ll_{\varepsilon} T^{1 / 2+2 \varepsilon}+\kappa_{j}^{1+2 \varepsilon},
$$

providing that the subinterval in question is not empty. If (2.6) fails to hold, then $t^{2}<\frac{1}{2} \kappa_{j}^{2}$ holds. Hence $\left|t^{2}-\kappa_{j}^{2}\right|<\frac{3}{2} \kappa_{j}^{2}$ and

$$
F^{\prime}(t) \geq \log \frac{4 \pi^{2} \kappa_{j}^{2}}{\left|t^{2}-\kappa_{j}^{2}\right|}>\log \frac{8 \pi^{2}}{3}>3 .
$$


Therefore, by the first derivative test (Lemma 2.1 of [8]), the bound in (2.7) certainly holds for the corresponding portion of the integral over $\left[T_{1}, T_{2}\right]$. Hence we obtain

$$
\sum_{2} \ll_{\varepsilon}\left(T^{1 / 2+2 \varepsilon}+\kappa_{j}^{1+2 \varepsilon}\right) \sum_{n>\kappa_{j}^{2} / x}\left|t_{j}(n)\right| n^{-\varepsilon-1} \ll_{\varepsilon}\left(T^{1 / 2}+\kappa_{j}\right) x^{3 \varepsilon} .
$$

From (2.3), (2.4) and (2.8) we obtain

$$
\sum_{n \leq x} t_{j}(n) \ll_{\varepsilon}\left(\kappa_{j}+x^{1+\alpha} T^{-1}+T^{1 / 2}\right) x^{3 \varepsilon} \ll_{\varepsilon}\left(\kappa_{j}+x^{(1+\alpha) / 3}\right) x^{3 \varepsilon}
$$

if $T=x^{(2+2 \alpha) / 3}$. Since $x^{(1+\alpha) / 3} \leq \kappa_{j}^{(2+2 \alpha) / 3} \leq \kappa_{j}^{5 / 6}$, Theorem 1 follows from (2.9).

3. Some auxiliary results. In this section we lay the groundwork for the proof of Theorem 2, and state five lemmas. In the next section we shall obtain a suitable explicit formula for $T_{j}(x)$ that will be squared and integrated termwise to produce Theorem 2 . It would be possible to treat also the case $\kappa_{j}>X^{1 / 2}$, but the difficulties involving the transitional region of the $K$-Bessel function terms would be overwhelming, and for this reason we shall be working with the condition $\kappa_{j} \leq X^{1 / 2}$. Such a condition, however, is not necessary for this section, except in Lemma 2.

Lemma 2. Let $1 \ll N \ll x, \kappa_{j} \leq x^{1 / 2}$. Then for any given $\varepsilon>0$ we have uniformly

$$
\begin{aligned}
T_{j}(x)= & \frac{x^{1 / 4}}{\pi \sqrt{2}} \sum_{n \leq N} t_{j}(n)\left(n+\frac{\kappa_{j}^{2}}{4 \pi^{2} x}\right)^{-3 / 4} \sin G_{\kappa_{j}}(4 \pi \sqrt{n x}) \\
& +H_{j}(0)+O_{\varepsilon}\left(x^{1 / 2+\alpha+\varepsilon} N^{-1 / 2}\right),
\end{aligned}
$$

where $\alpha$ is the constant in (1.7) and

$$
\begin{aligned}
G_{\kappa}(u): & =\left(u^{2}+4 \kappa^{2}\right)^{1 / 2}-2 \kappa \operatorname{arsh}\left(\frac{2 \kappa}{u}\right)+\frac{\pi}{4}, \\
& \operatorname{arsh} u=\log \left(u+\left(u^{2}+1\right)^{1 / 2}\right) .
\end{aligned}
$$

Lemma 3. For $x>0$ we have

$$
\begin{aligned}
T_{j}(x)= & \frac{\pi i}{\operatorname{sh}\left(\pi \kappa_{j}\right)} \sum_{n=1}^{\infty} t_{j}(n) \int_{0}^{x}\left(J_{2 i \kappa_{j}}(4 \pi \sqrt{n v})-J_{-2 i \kappa_{j}}(4 \pi \sqrt{n v})\right) d v \\
& +4 \varepsilon_{j} \operatorname{ch}\left(\pi \kappa_{j}\right) \sum_{n=1}^{\infty} t_{j}(n) \int_{0}^{x} K_{2 i \kappa_{j}}(4 \pi \sqrt{n v}) d v
\end{aligned}
$$

The series occurring in (3.3) are boundedly convergent for $x$ lying in any fixed interval $\left[x_{1}, x_{2}\right] \subset(0, \infty)$. 
The proofs of Lemmas 2 and 3 are found in [20]. For the definitions and relevant properties of the $J$ - and $K$-Bessel functions the reader is referred to the monograph of G. N. Watson [31].

Lemma 4. For $x>0$ and $\kappa \gg 1$ we have

$$
\begin{aligned}
& \frac{-J_{2 i \kappa}(x)+J_{-2 i \kappa}(x)}{\sin (i \pi \kappa)} \\
& =2 \pi^{-1 / 2}\left(\frac{x^{2}}{4}+\kappa^{2}\right)^{-1 / 4} \cos G_{\kappa}(x) \\
& \quad+\left(\frac{x^{2}}{4}+\kappa^{2}\right)^{-3 / 4}\left(a^{+}(x, \kappa) e^{i G_{\kappa}(x)}+a^{-}(x, \kappa) e^{-i G_{\kappa}(x)}\right) \\
& \quad+O\left(\left(x^{2}+\kappa^{2}\right)^{-5 / 4}\right),
\end{aligned}
$$

where $G_{\kappa}(x)$ is given by (3.2) and

$$
a^{ \pm}(x, \kappa) \ll 1, \quad \frac{\partial}{\partial x} a^{ \pm}(x, \kappa) \ll 1 / x .
$$

This is deduced from the well-known asymptotic formulas for the $J$-Bessel function (see A. Erdélyi et al. [6] or T. Meurman [20]). We shall also need two lemmas on integrals with the $J$-Bessel function. Henceforth we let for brevity

$$
J:=\frac{-J_{2 i \kappa}(u)+J_{-2 i \kappa}(u)}{\sin (i \pi \kappa)}, \quad K:=\frac{u^{2}}{4}+\kappa^{2} .
$$

Then we have

LEMma 5. For $\kappa \gg 1$ the integral

$$
I:=\int_{0}^{\infty}\left\{u J-\pi^{-1 / 2}\left(u^{2} K^{-3 / 4} \sin G_{\kappa}(u)\right)^{\prime}\right\} d u
$$

is convergent, and moreover,

$$
I \ll \kappa^{-1 / 2} .
$$

Proof. We have

$$
\begin{gathered}
\left(u^{2} K^{-3 / 4} \sin G_{\kappa}(u)\right)^{\prime}=2 u K^{-3 / 4} \sin G_{\kappa}(u)+u^{2}\left(K^{-3 / 4} \sin G_{\kappa}(u)\right)^{\prime}, \\
G_{\kappa}^{\prime}(u)=2 u^{-1} K^{1 / 2} .
\end{gathered}
$$

Therefore by the first derivative test $\int_{0}^{\infty} u K^{-3 / 4} \sin G_{\kappa}(u) d u \ll \kappa^{-1 / 2}$, and it remains to show that $I=I(1)$ converges and that $I(1) \ll \kappa^{-1 / 2}$, where we set

$$
I(s):=\int_{0}^{\infty} u^{s}\left\{J-\pi^{-1 / 2} u\left(K^{-3 / 4} \sin G_{\kappa}(u)\right)^{\prime}\right\} d u .
$$


In fact, $I(s)$ is holomorphic for $0<\operatorname{Re} s \leq 1$. Namely, by using (3.9) one can write $I(s)=I_{1}(s)+I_{2}(s)$, where

$$
\begin{aligned}
& I_{1}(s)=\frac{3}{8 \sqrt{\pi}} \int_{0}^{\infty} u^{s+2} K^{-7 / 4} \sin G_{\kappa}(u) d u, \\
& I_{2}(s)=\int_{0}^{\infty} u^{s}\left\{J-2 \pi^{-1 / 2} K^{-1 / 4} \cos G_{\kappa}(u)\right\} d u .
\end{aligned}
$$

The holomorphy of $I_{1}(s)$ for $0<\operatorname{Re} s \leq 1$ follows then by the first derivative test, and that of $I_{2}(s)$ by Lemma 4 .

Let now $0<\operatorname{Re} s<1 / 2$. By using the Mellin transform formula

$$
\int_{0}^{\infty} J_{\nu}(x) x^{s-1} d x=\frac{2^{s-1} \Gamma((s+\nu) / 2)}{\Gamma((\nu-s) / 2+1)} \quad(-\operatorname{Re} \nu<\operatorname{Re} s<3 / 2)
$$

it follows that

$$
\int_{0}^{\infty} u^{s} J d u=-\pi^{-1} 2^{s+1} \sin \left(\frac{\pi s}{2}\right) \Gamma\left(\frac{s+1}{2}+i \kappa\right) \Gamma\left(\frac{s+1}{2}-i \kappa\right) .
$$

Integration by parts yields

$$
\int_{0}^{\infty} u^{s+1}\left(K^{-3 / 4} \sin G_{\kappa}(u)\right)^{\prime} d u=-(s+1) \int_{0}^{\infty} u^{s} K^{-3 / 4} \sin G_{\kappa}(u) d u
$$

and the integral on the right-hand side of (3.11) is, by the first derivative test, holomorphic for $0<\operatorname{Re} s \leq 1$. Hence by analytic continuation we infer from (3.10) and (3.11) that

$$
I(1)=-4 \pi^{-1}|\Gamma(1+i \kappa)|^{2}+2 \pi^{-1 / 2} \int_{0}^{\infty} u K^{-3 / 4} \sin G_{\kappa}(u) d u .
$$

By the first derivative test it is seen that the above integral converges and is $\ll \kappa^{-1 / 2}$. This proves the lemma.

Lemma 6. For $\kappa \gg 1$,

$$
\int_{U}^{\infty}\left\{u J-\pi^{-1 / 2}\left(u^{2} K^{-3 / 4} \sin G_{\kappa}(u)\right)^{\prime}\right\} d u \ll\left(U^{2}+\kappa^{2}\right)^{-1 / 4} .
$$

Proof. We note that by (3.8) and (3.9),

$$
\begin{aligned}
\left(u^{2} K^{-3 / 4} \sin G_{\kappa}(u)\right)^{\prime}= & 2 u K^{-3 / 4} \sin G_{\kappa}(u) \\
& -\frac{3}{8} u^{3} K^{-7 / 4} \sin G_{\kappa}(u)+2 u K^{-1 / 4} \cos G_{\kappa}(u),
\end{aligned}
$$

and we insert this expression in (3.12). The third term is cancelled by the 
main term given by Lemma 4 . Hence it remains to show that

$$
\begin{gathered}
\left|\int_{U}^{\infty} u K^{-3 / 4} \sin G_{\kappa}(u) d u\right|+\left|\int_{U}^{\infty} u^{3} K^{-7 / 4} \sin G_{\kappa}(u) d u\right| \\
\ll\left(U^{2}+\kappa^{2}\right)^{-1 / 4}, \\
\int_{U}^{\infty} u K^{-3 / 4} a^{ \pm}(u, \kappa) e^{ \pm i G_{\kappa}(u)} d u \ll\left(U^{2}+\kappa^{2}\right)^{-1 / 4}
\end{gathered}
$$

and

$$
\int_{U}^{\infty} u K^{-5 / 4} d u \ll\left(U^{2}+\kappa^{2}\right)^{-1 / 4}
$$

The bound in (3.13) follows from the first derivative test, from the one in (3.14) by an integration by parts and from (3.5), and the one given by (3.15) is trivial.

4. The explicit formula for $T_{j}(x)$. In this section we derive an explicit formula for $T_{j}(x)$, which is suitable for the proof of Theorem 2. We start from (3.3) of Lemma 3.

By using Lemmas 5 and $6\left(G_{\kappa}(u)\right.$ is defined by $\left.(3.2)\right)$ we find that

$$
\begin{aligned}
& \frac{\pi i}{\operatorname{sh}(\pi \kappa)} \int_{0}^{x}\left\{J_{2 i \kappa}(4 \pi \sqrt{n v})-J_{-2 i \kappa}(4 \pi \sqrt{n v})\right\} d v \\
& =2 \sqrt{\pi} x\left(4 \pi^{2} n x+\kappa^{2}\right)^{-3 / 4} \sin G_{\kappa}(4 \pi \sqrt{n x}) \\
& \quad+\frac{1}{8 \pi n} \int_{0}^{4 \pi \sqrt{n x}}\left\{u \frac{J_{2 i \kappa}(u)-J_{-2 i \kappa}(u)}{-i \operatorname{sh}(\pi \kappa)}\right. \\
& =2 \sqrt{\pi} x\left(4 \pi^{2} n x+\kappa^{2}\right)^{-3 / 4} \sin G_{\kappa}(4 \pi \sqrt{n x}) \\
& \left.\quad+\frac{1}{8 \pi n} \int_{0}^{\infty}\left\{u \frac{J_{2 i \kappa}(u)-J_{-2 i \kappa}(u)}{-i \operatorname{sh}(\pi \kappa)} \sin G_{\kappa}(u)\right)^{\prime}\right\} d u \\
& \left.\quad-\frac{1}{\sqrt{\pi}}\left(u^{2}\left(\frac{u^{2}}{4}+\kappa^{2}\right)^{-3 / 4} \sin G_{\kappa}(u)\right)^{\prime}\right\} d u \\
& +O\left(n^{-1}\left(n x+\kappa^{2}\right)^{-1 / 4}\right) .
\end{aligned}
$$


To transform the second series in (3.3) note first that

$$
\int_{0}^{\infty} K_{2 i \kappa}(4 \pi \sqrt{n v}) d v=\frac{|\Gamma(1+i \kappa)|^{2}}{8 \pi^{2} n} .
$$

Hence

$$
\begin{aligned}
& 4 \varepsilon_{j} \operatorname{ch}\left(\pi \kappa_{j}\right) \int_{0}^{x} K_{2 i \kappa_{j}}(4 \pi \sqrt{n v}) d v \\
= & -4 \varepsilon_{j} \operatorname{ch}\left(\pi \kappa_{j}\right) \int_{x}^{\infty} K_{2 i \kappa_{j}}(4 \pi \sqrt{n v}) d v+\frac{1}{2 \pi^{2} n} \varepsilon_{j} \operatorname{ch}\left(\pi \kappa_{j}\right)\left|\Gamma\left(1+i \kappa_{j}\right)\right|^{2} \\
= & -4 \varepsilon_{j} \operatorname{ch}\left(\pi \kappa_{j}\right) \int_{x}^{\infty} K_{2 i \kappa_{j}}(4 \pi \sqrt{n v}) d v+\left\{X_{j}(0)+\frac{1}{2 \pi^{2}}\left|\Gamma\left(1+i \kappa_{j}\right)\right|^{2}\right\} \frac{1}{n},
\end{aligned}
$$

where $X_{j}(s)$ is given by (1.3). At this point we recall that (see G. N. Watson [31])

$$
K_{2 i \kappa_{j}}(x)=\frac{1}{2 \operatorname{ch}\left(\pi \kappa_{j}\right)} \int_{-\infty}^{\infty} \cos (x \operatorname{sh} t) \cos \left(2 \kappa_{j} t\right) d t
$$

In the above integral write

$$
\cos (x \operatorname{sh} t)=\operatorname{Re}\left(e^{i x \operatorname{sh} t}\right)
$$

and shift the line of integration to $\operatorname{Im} t=\pi / 2$. Since

$$
\operatorname{sh}(t+\pi i / 2)=i \operatorname{ch} t
$$

we obtain

$$
K_{2 i \kappa_{j}}(x) \ll \int_{0}^{\infty} e^{-x \operatorname{ch} t} d t \leq \int_{0}^{\infty} e^{-x\left(1+t^{2} / 2\right)} d t=\left(\frac{\pi}{2 x}\right)^{1 / 2} e^{-x}
$$

uniformly in $\kappa_{j}$. At this point we suppose that $X \leq x \leq Y \leq 2 X$, and make use of the condition $\kappa_{j} \leq X^{1 / 2}$, required for Theorem 2 . Then by using (4.3) we have

$$
\begin{aligned}
\operatorname{ch}\left(\pi \kappa_{j}\right) \int_{x}^{\infty} K_{2 i \kappa_{j}}(4 \pi \sqrt{n v}) d v & \ll e^{\pi \kappa_{j}} \int_{x}^{\infty}(n v)^{-1 / 4} e^{-4 \pi \sqrt{n v}} d v \\
& \ll e^{\pi \kappa_{j}} n^{-1} \int_{4 \pi \sqrt{n x}}^{\infty} u^{1 / 2} e^{-u} d u \\
& \ll n^{-1}(n x)^{1 / 4} e^{\pi \kappa_{j}-4 \pi \sqrt{n x}} \\
& \ll n^{-1}\left(n x+\kappa_{j}^{2}\right)^{-1 / 4},
\end{aligned}
$$


which is the same as the error term in (4.1). Thus (4.2) gives

$$
\begin{aligned}
4 \varepsilon_{j} \operatorname{ch}\left(\pi \kappa_{j}\right) & \int_{0}^{x} K_{2 i \kappa_{j}}(4 \pi \sqrt{n v}) d v \\
= & \left\{X_{j}(0)+\frac{1}{2 \pi^{2}}\left|\Gamma\left(1+i \kappa_{j}\right)\right|^{2}\right\} \frac{1}{n}+O\left(\frac{1}{n}\left(n x+\kappa_{j}^{2}\right)^{-1 / 4}\right) .
\end{aligned}
$$

We insert (4.1) and (4.4) in (3.3), noting that by the functional equation (1.2),

$$
X_{j}(0) \sum_{n=1}^{\infty} t_{j}(n) n^{-1}=X_{j}(0) H_{j}(1)=H_{j}(0)
$$

and using (see (1.13))

$$
\sum_{n=1}^{\infty}\left|t_{j}(n)\right| n^{-1}\left(n x+\kappa_{j}^{2}\right)^{-1 / 4} \ll_{\varepsilon} \kappa_{j}^{\varepsilon} x^{-1 / 4} .
$$

The resulting formula is contained in

Lemma 7. Suppose $\kappa_{j} \leq X^{1 / 2}$ and $X \leq x \leq Y \leq 2 X$, let $G_{\kappa}(u)$ be given by (3.2), and let

$$
\begin{aligned}
& S_{j}(x):=\frac{x^{1 / 4}}{\pi \sqrt{2}} \sum_{n=1}^{\infty} t_{j}(n)\left(n+\frac{1}{x}\left(\frac{\kappa_{j}^{2}}{2 \pi}\right)\right)^{-3 / 4} \sin G_{\kappa_{j}}(4 \pi \sqrt{n x}) \\
& h(\kappa):=\left\{\frac{|\Gamma(1+i \kappa)|^{2}}{2 \pi^{2}}+\frac{1}{8 \pi} \int_{0}^{\infty}\left(u \frac{J_{2 i \kappa}(u)-J_{-2 i \kappa}(u)}{-i \operatorname{sh}(\pi \kappa)}\right.\right. \\
&\left.\left.-\pi^{-1 / 2}\left(u^{2}\left(\frac{u^{2}}{4}+\kappa^{2}\right)^{-3 / 4} \sin G_{\kappa}(u)\right)^{\prime}\right) d u\right\} H_{j}(1) .
\end{aligned}
$$

Then we have, uniformly in $\kappa_{j}$,

$$
T_{j}(x)=S_{j}(x)+H_{j}(0)+h\left(\kappa_{j}\right)+O_{\varepsilon}\left(\kappa_{j}^{\varepsilon} X^{-1 / 4}\right) .
$$

We shall now further transform the above formula for $T_{j}(x)$, but we wish to note here that by Lemma 5 ,

$$
h\left(\kappa_{j}\right) \ll_{\varepsilon} \kappa_{j}^{\varepsilon-1 / 2},
$$

since $H_{j}(1) \ll_{\varepsilon} \kappa_{j}^{\varepsilon}$. Thus $h\left(\kappa_{j}\right)$ is the "small" constant appearing in (4.7), whereas $H_{j}(0)$ may be considered "large", in view of

$$
\left|H_{j}(0)\right|=\left|H_{j}(1) X_{j}(0)\right| \gg \kappa_{j}\left|H_{j}(1)\right| .
$$

Our goal is to truncate the series $S_{j}(x)$ and estimate the resulting error. 
Let for brevity $q=\left(\kappa_{j} /(2 \pi)\right)^{2}$ and consider

$$
V=V\left(x ; M, \kappa_{j}\right):=x^{1 / 4} \sum_{n>M} t_{j}(n)\left(n+\frac{q}{x}\right)^{-3 / 4} \sin G_{\kappa_{j}}(4 \pi \sqrt{n x}),
$$

where $M \geq x^{3}$, so that $\kappa_{j} \leq X^{1 / 2} \leq x^{1 / 2} \leq M^{1 / 6}$. By partial summation and (1.6) it follows that

$$
\begin{aligned}
V= & -x^{1 / 4} \int_{M}^{\infty}\left(\sum_{n \leq y} t_{j}(n)\right)\left\{(y+q / x)^{-3 / 4} \sin G_{\kappa_{j}}(4 \pi \sqrt{y x})\right\}^{\prime} d y \\
& -x^{1 / 4}\left(\sum_{n \leq M} t_{j}(n)\right)(M+q / x)^{-3 / 4} \sin G_{\kappa_{j}}(4 \pi \sqrt{M x}) \\
= & -2 \pi x^{3 / 4} \int_{M}^{\infty}\left(\sum_{n \leq y} t_{j}(n)\right) y^{-1}(y+q / x)^{-1 / 4} \cos G_{\kappa_{j}}(4 \pi \sqrt{y x}) d y \\
& +O_{\varepsilon}\left(x^{1 / 4} M^{-1 / 4}\right) \\
= & -2 \pi x^{3 / 4} \sum_{k=0}^{\infty} I\left(2^{k} M\right)+O_{\varepsilon}\left(x^{1 / 4} M^{-1 / 4}\right),
\end{aligned}
$$

where

$$
I(W):=\int_{W}^{2 W}\left(\sum_{n \leq y} t_{j}(n)\right) y^{-1}(y+q / x)^{-1 / 4} \cos G_{\kappa_{j}}(4 \pi \sqrt{y x}) d y .
$$

We replace the sum in (4.10) by the expression given by Lemma 2, namely

$$
\begin{aligned}
\sum_{n \leq y} t_{j}(n)= & \frac{y^{1 / 4}}{\pi \sqrt{2}} \sum_{n \leq W} t_{j}(n)(n+q / y)^{-3 / 4} \sin G_{\kappa_{j}}(4 \pi \sqrt{n y}) \\
& +H_{j}(0)+O_{\varepsilon}\left(W^{\alpha+\varepsilon}\right) \quad(W \leq y \leq 2 W),
\end{aligned}
$$

where $\alpha \leq 1 / 5$. Since $H_{j}(0) \ll_{\varepsilon} \kappa_{j}^{1+\varepsilon}$, we obtain

$$
\begin{aligned}
I(W)= & \frac{1}{\pi \sqrt{2}} \sum_{n \leq W} t_{j}(n) \int_{W}^{2 W}(y+q / x)^{-1 / 4}(n y+q)^{-3 / 4} \\
& \times \cos G_{\kappa_{j}}(4 \pi \sqrt{x y}) \sin G_{\kappa_{j}}(4 \pi \sqrt{n y}) d y \\
& +O_{\varepsilon}\left(\left(\kappa_{j}^{1+\varepsilon}+W^{\alpha+\varepsilon}\right) W^{-1 / 4}\right) .
\end{aligned}
$$

To evaluate the above integral we let

$$
\Psi(y):=G_{\kappa_{j}}(4 \pi \sqrt{y x}) \pm G_{\kappa_{j}}(4 \pi \sqrt{y n}),
$$


so that

$$
\begin{aligned}
\left|\Psi^{\prime}(y)\right| & =\left|\frac{4 \pi^{2}(x-n)}{\left(4 \pi^{2} y x+\kappa_{j}^{2}\right)^{1 / 2} \mp\left(4 \pi^{2} y n+\kappa_{j}^{2}\right)^{1 / 2}}\right| \\
& \gg \begin{cases}(x / y)^{1 / 2} & \text { if } n \leq x / 2, \\
|x-n|(x y)^{-1 / 2} & \text { if } x / 2<n \leq 2 x, \\
(n / y)^{1 / 2} & \text { if } n>2 x .\end{cases}
\end{aligned}
$$

Hence by the first derivative test

$$
\begin{aligned}
\int_{W}^{2 W}(y+q / x)^{-1 / 4}(n y & +q)^{-3 / 4} e^{i \Psi(y)} d y \\
& \ll \begin{cases}n^{-3 / 4} W^{-1 / 2} x^{-1 / 2} & \text { if } n \leq x / 2, \\
x^{-1 / 4} W^{-1 / 2}|x-n|^{-1} & \text { if } x / 2<n \leq 2 x, \\
n^{-5 / 4} W^{-1 / 2} & \text { if } n>2 x .\end{cases}
\end{aligned}
$$

This gives

$$
\begin{aligned}
I(W) \ll & \sum_{n \leq x / 2}\left|t_{j}(n)\right| n^{-3 / 4} W^{-1 / 2} x^{-1 / 2} \\
& +\sum_{x / 2<n \leq 2 x,|n-x| \geq 1 / 2}\left|t_{j}(n)\right| x^{-1 / 4} W^{-1 / 2}|x-n|^{-1} \\
& +\sum_{n \geq 2 x}\left|t_{j}(n)\right| n^{-5 / 4} W^{-1 / 2} \\
& +\min \left(x^{-1 / 4} W^{-1 / 2}\|x\|^{-1}, x^{-3 / 4}\right)\left|t_{j}\left(n_{x}\right)\right| \\
& +\left(\kappa_{j}^{1+\varepsilon}+W^{\alpha+\varepsilon}\right) W^{-1 / 4},
\end{aligned}
$$

where $\|u\|$ is the distance from $u$ to the nearest integer, and $n_{x}$ is the integer satisfying $\left|n_{x}-x\right|<1 / 2$, if it exists. All three sums above are easily seen to be $\ll W^{-1 / 2}$, and moreover,

$\min \left(x^{-1 / 4} W^{-1 / 2}\|x\|^{-1}, x^{-3 / 4}\right)\left|t_{j}\left(n_{x}\right)\right| \ll \min \left(W^{-1 / 2}\|x\|^{-1}, 1\right)$,
$\left(\kappa_{j}^{1+\varepsilon}+W^{\alpha+\varepsilon}\right) W^{-1 / 4} \leq\left(M^{1 / 6+\varepsilon}+W^{\alpha+\varepsilon}\right) W^{-1 / 4} \leq 2 W^{\alpha+\varepsilon-1 / 4} \leq W^{-1 / 25}$ for $W \geq W_{0}(\varepsilon)$ if $0<\varepsilon<1 / 100$. Altogether we certainly have, for $W \geq M$,

$$
I(W) \ll \min \left(W^{-1 / 25}\|x\|^{-1}, 1\right) .
$$

Let now

$$
k_{0}:=\max \left(0, \frac{\log \left(M^{-1}\|x\|^{-25}\right)}{\log 2}\right) .
$$


By using (4.11) we have, since $M^{-1 / 4} \leq x^{-3 / 4}$,

$$
\begin{aligned}
V & \ll x^{3 / 4} \sum_{0 \leq k \leq k_{0}}\left|I\left(2^{k} M\right)\right|+x^{3 / 4} \sum_{k \geq k_{0}}\left|I\left(2^{k} M\right)\right|+x^{1 / 4} M^{-1 / 4} \\
& \ll k_{0} x^{3 / 4}+x^{3 / 4}\|x\|^{-1} \sum_{k \geq k_{0}}\left(2^{k} M\right)^{-1 / 25}+x^{-1 / 2} \\
& \ll k_{0} x^{3 / 4}+x^{3 / 4}\|x\|^{-1}\left(2^{k_{0}} M\right)^{-1 / 25}+x^{-1 / 4} .
\end{aligned}
$$

It follows that

$$
V \ll \begin{cases}x^{-1 / 4} & \text { if }\|x\|>x M^{-1 / 25}, \\ x^{3 / 4} \log (1 /\|x\|) & \text { if }\|x\| \leq x M^{-1 / 25} .\end{cases}
$$

Indeed, in the first case $k_{0}=0$ and we get $V \ll x^{-1 / 4}$. But in any case

so that

$$
\frac{\log \left(M^{-1}\|x\|^{-25}\right)}{\log 2} \leq k_{0} \ll \log \frac{1}{\|x\|}
$$

$V \ll x^{3 / 4} \log \frac{1}{\|x\|}+x^{3 / 4}\|x\|^{-1}\left(M^{-1}\|x\|^{-25} M\right)^{-1 / 25}+x^{-1 / 4} \ll x^{3 / 4} \log \frac{1}{\|x\|}$.

Thus finally from Lemma 7, (4.9) and (4.12) we obtain the desired expression for $T_{j}(x)$. This is

Lemma 8. Suppose $\kappa_{j} \leq X^{1 / 2}, M \geq x^{3}$ and $X \leq x \leq Y \leq 2 X$, and let $G_{\kappa}(u)$ be given by (3.2) and $h(\kappa)$ by (4.6). Then

$$
\begin{aligned}
T_{j}(x)= & \frac{x^{1 / 4}}{\pi \sqrt{2}} \sum_{n \leq M} t_{j}(n)\left(n+\frac{1}{x}\left(\frac{\kappa_{j}}{2 \pi}\right)^{2}\right)^{-3 / 4} \sin G_{\kappa_{j}}(4 \pi \sqrt{n x}) \\
& +H_{j}(0)+h\left(\kappa_{j}\right)+R_{j}(x),
\end{aligned}
$$

where uniformly

$$
R_{j}(x) \ll_{\varepsilon} \begin{cases}\kappa_{j}^{\varepsilon} x^{-1 / 4} & \text { if }\|x\|>x M^{-1 / 25} \\ x^{3 / 4} \log (1 /\|x\|) & \text { if }\|x\| \leq x M^{-1 / 25}\end{cases}
$$

5. Proof of Theorem 2. We can now use the fundamental Lemma 8 to prove Theorem 2. Suppose $X<Y \leq 2 X, \kappa_{j} \leq X^{1 / 2}$, choose $M=X^{100}$ in (4.13), (4.14), and consider

$$
F_{j}=F_{j}(X, Y):=\int_{X}^{Y}\left(T_{j}(x)-H_{j}(0)-h\left(\kappa_{j}\right)\right)^{2} d x .
$$

Setting

$$
W_{j}(x):=\frac{x^{1 / 4}}{\pi \sqrt{2}} \sum_{n \leq M} t_{j}(n)\left(n+\frac{1}{x}\left(\frac{\kappa_{j}}{2 \pi}\right)^{2}\right)^{-3 / 4} \sin G_{\kappa_{j}}(4 \pi \sqrt{n x})
$$


we have

$$
F_{j}=\int_{X}^{Y} W_{j}^{2}(x) d x+2 \int_{X}^{Y} W_{j}(x) R_{j}(x) d x+\int_{X}^{Y} R_{j}^{2}(x) d x .
$$

We have by (4.14),

$$
\int_{X}^{Y} R_{j}^{2}(x) d x \ll_{\varepsilon} \kappa_{j}^{\varepsilon} X^{1 / 2}+X^{3 / 2} \int_{\|x\| \ll X^{-3}}\left(\log \frac{1}{\|x\|}\right)^{2} d x \ll_{\varepsilon} X^{1 / 2+\varepsilon} .
$$

We write

$$
\int_{X}^{Y} W_{j}^{2}(x) d x=F_{j 1}+F_{j 2}
$$

say, where

$$
\begin{aligned}
F_{j 1}= & \frac{1}{2 \pi^{2}} \sum_{m, n \leq M ; m \neq n} t_{j}(m) t_{j}(n) \int_{X}^{Y} x^{1 / 2}\left(m+\frac{1}{x}\left(\frac{\kappa_{j}}{2 \pi}\right)^{2}\right)^{-3 / 4} \\
& \times\left(n+\frac{1}{x}\left(\frac{\kappa_{j}}{2 \pi}\right)^{2}\right)^{-3 / 4} \sin G_{\kappa_{j}}(4 \pi \sqrt{m x}) \sin G_{\kappa_{j}}(4 \pi \sqrt{n x}) d x, \\
F_{j 2}= & \frac{1}{2 \pi^{2}} \sum_{n \leq M} t_{j}^{2}(n) \int_{X}^{Y} x^{1 / 2}\left(n+\frac{1}{x}\left(\frac{\kappa_{j}}{2 \pi}\right)^{2}\right)^{-3 / 2} \sin ^{2} G_{\kappa_{j}}(4 \pi \sqrt{n x}) d x .
\end{aligned}
$$

Consider first $F_{j 1}$. In view of (3.9) we have

$$
\left|\frac{\partial}{\partial x}\left(G_{\kappa_{j}}(4 \pi \sqrt{m x}) \pm G_{\kappa_{j}}(4 \pi \sqrt{n x})\right)\right| \gg \frac{|m-n|}{\sqrt{x}(\sqrt{m}+\sqrt{n})},
$$

and consequently by the first derivative test

$$
\int_{X}^{Y} \ll X(m n)^{-3 / 4}\left(m^{1 / 2}+n^{1 / 2}\right)|m-n|^{-1} .
$$

Hence

$$
\begin{aligned}
F_{j 1} \ll & X \sum_{m<n \leq M}\left|t_{j}(m) t_{j}(n)\right| m^{-3 / 4} n^{-1 / 4}(n-m)^{-1} \\
= & X \sum_{n \leq M} \sum_{m \leq n / 2}\left|t_{j}(m) t_{j}(n)\right| \frac{m^{-3 / 4} n^{-1 / 4}}{n-m} \\
& +X \sum_{n \leq M} \sum_{n / 2<m<n}\left|t_{j}(m) t_{j}(n)\right| \frac{m^{-3 / 4} n^{-1 / 4}}{n-m} .
\end{aligned}
$$


Trivially we have

$$
\begin{aligned}
\sum_{n \leq M} \sum_{m \leq n / 2}\left|t_{j}(m) t_{j}(n)\right| & \frac{m^{-3 / 4} n^{-1 / 4}}{n-m} \\
& \ll \sum_{n \leq M}\left|t_{j}(n)\right| n^{-5 / 4} \sum_{m \leq n / 2}\left|t_{j}(m)\right| m^{-3 / 4} \ll_{\varepsilon} X^{\varepsilon} .
\end{aligned}
$$

By writing $m=n-k$ and using $|a b| \leq \frac{1}{2}\left(a^{2}+b^{2}\right)$ we obtain

$$
\begin{aligned}
\sum_{n \leq M} & \sum_{n / 2<m<n}\left|t_{j}(m) t_{j}(n)\right| \frac{m^{-3 / 4} n^{-1 / 4}}{n-m} \\
& \ll \sum_{n \leq M}\left|t_{j}(n)\right| n^{-1} \sum_{1 \leq k<n / 2}\left|t_{j}(n-k)\right| k^{-1} \\
& \ll \log M \cdot \max _{M_{1} \leq M} M_{1}^{-1} \sum_{k \leq M_{1} / 2} k^{-1} \sum_{\max \left(2 k, M_{1} / 2\right)<n \leq M_{1}}\left|t_{j}(n) t_{j}(n-k)\right| \\
& \ll \log M \cdot \max _{M_{1} \leq M} M_{1}^{-1} \sum_{k \leq M_{1} / 2} k^{-1} \sum_{n \leq M_{1}} t_{j}^{2}(n) \ll X^{\varepsilon},
\end{aligned}
$$

where (1.13) was used. Thus

$$
F_{j 1} \ll_{\varepsilon} X^{1+\varepsilon} .
$$

It remains to consider $F_{j 2}$. We use

$$
\sin ^{2} G_{\kappa_{j}}(4 \pi \sqrt{n x})=\frac{1}{2}\left(1-\cos 2 G_{\kappa_{j}}(4 \pi \sqrt{n x})\right)
$$

and estimate the cosine terms by the first derivative test. This gives

$$
F_{j 2}=\frac{1}{4 \pi^{2}} \sum_{n \leq M} t_{j}^{2}(n) \int_{X}^{Y} x^{1 / 2}\left(n+\frac{1}{x}\left(\frac{\kappa_{j}}{2 \pi}\right)^{2}\right)^{-3 / 2} d x+O_{\varepsilon}\left(X^{1+\varepsilon}\right) .
$$

Since $M=X^{100}$ we obtain

$$
\begin{aligned}
\sum_{n>M} t_{j}^{2}(n) \int_{X}^{Y} x^{1 / 2}\left(n+\frac{1}{x}\left(\frac{\kappa_{j}}{2 \pi}\right)^{2}\right)^{-3 / 2} d x & \ll X^{3 / 2} \sum_{n>M} t_{j}^{2}(n) n^{-3 / 2} \\
& \ll_{\varepsilon} X^{3 / 2} \kappa_{j}^{\varepsilon} M^{-1 / 2} \ll 1,
\end{aligned}
$$

and consequently

$$
F_{j 2}=\frac{1}{4 \pi^{2}} \sum_{n=1}^{\infty} t_{j}^{2}(n) \int_{X}^{Y} x^{1 / 2}\left(n+\frac{1}{x}\left(\frac{\kappa_{j}}{2 \pi}\right)^{2}\right)^{-3 / 2} d x+O_{\varepsilon}\left(X^{1+\varepsilon}\right) .
$$


Thus from (5.3)-(5.5) it follows that

$$
\begin{aligned}
& \int_{X}^{Y} W_{j}^{2}(x) d x \\
& \quad=\frac{1}{4 \pi^{2}} \sum_{n=1}^{\infty} t_{j}^{2}(n) \int_{X}^{Y} x^{1 / 2}\left(n+\frac{1}{x}\left(\frac{\kappa_{j}}{2 \pi}\right)^{2}\right)^{-3 / 2} d x+O_{\varepsilon}\left(X^{1+\varepsilon}\right),
\end{aligned}
$$

and the main term on the right-hand side of (5.6) is $\ll_{\varepsilon} \kappa_{j}^{\varepsilon} X^{3 / 2} \ll_{\varepsilon} X^{3 / 2+\varepsilon}$. By (5.2), (5.6) and the Cauchy-Schwarz inequality we obtain

$$
\int_{X}^{Y} W_{j}(x) R_{j}(x) d x \ll_{\varepsilon} X^{1+\varepsilon},
$$

and (5.1) gives then (1.16).

To obtain (1.17) from (1.16) write first

$$
\begin{aligned}
\int_{X}^{2 X} T_{j}^{2}(x) d x= & \int_{X}^{2 X}\left(T_{j}(x)-H_{j}(0)-h\left(\kappa_{j}\right)\right)^{2} d x \\
& +\left(H_{j}(0)+h\left(\kappa_{j}\right)\right)^{2} X \\
& +2\left(H_{j}(0)+h\left(\kappa_{j}\right)\right) \int_{X}^{2 X}\left(T_{j}(x)-H_{j}(0)-h\left(\kappa_{j}\right)\right) d x .
\end{aligned}
$$

The first integral on the right-hand side of (5.7) is evaluated by (1.16), if we note that, for $\kappa_{j} \leq X^{1 / 2}$ and $X \leq x \leq 2 X$,

$$
\left(n+\frac{1}{x}\left(\frac{\kappa_{j}}{2 \pi}\right)^{2}\right)^{-3 / 2}=n^{-3 / 2}\left(1+O\left(\frac{\kappa_{j}^{2}}{n X}\right)\right),
$$

and the contribution of the error term is

$$
\sum_{n=1}^{\infty} t_{j}^{2}(n) n^{-5 / 2} \kappa_{j}^{2} \int_{X}^{2 X} x^{1 / 2} X^{-1} d x \ll_{\varepsilon} \kappa_{j}^{2} X^{1 / 2+\varepsilon} .
$$

By Lemma 7 and the first derivative test we obtain

$$
\int_{X}^{2 X}\left(T_{j}(x)-H_{j}(0)-h\left(\kappa_{j}\right)\right) d x \ll_{\varepsilon} X^{3 / 4+\varepsilon} .
$$

Since $H_{j}(0) \ll_{\varepsilon} \kappa_{j}^{1+\varepsilon}$ and $h\left(\kappa_{j}\right) \ll_{\varepsilon} \kappa_{j}^{\varepsilon-1 / 2}$, we then obtain (1.17) from (5.7) and (5.8). 
6. Proof of Theorem 3. As stated in Section 1, N. V. Proskurin [27] investigated the summatory function of $\varrho_{j_{1}}(n) \overline{\varrho_{j_{2}}(n)}$, where $\kappa_{j_{1}}$ and $\kappa_{j_{2}}$ are not necessarily fixed. If $\kappa_{j_{1}}=\kappa_{j_{2}}=\kappa_{j}$, then his results are

$$
\begin{aligned}
\sum_{n \leq x} t_{j}^{2}(n) & =\frac{12}{\pi^{2} \alpha_{j}}+O_{\varepsilon}\left(x^{7 / 6+\varepsilon}+x^{1+\varepsilon} \kappa_{j}^{1 / 2}+x^{1 / 2} \kappa_{j}^{1+\varepsilon}\right) \\
\sum_{n \leq x} c_{j}(n)=2 x \operatorname{ch}\left(\pi \kappa_{j}\right)+O_{\varepsilon}\left(\alpha_{j} \operatorname{ch}(\right. & \left.\pi \kappa_{j}\right)\left(\kappa_{j}^{3 / 2+\varepsilon}+x^{19 / 18+\varepsilon}\right. \\
& \left.\left.+x^{7 / 10+\varepsilon} \kappa_{j}^{4 / 5}+x^{-1 / 2} \kappa_{j}^{2+\varepsilon}\right)\right),
\end{aligned}
$$

$$
\begin{aligned}
\sum_{n \leq x} t_{j}^{2}(n)= & \frac{12 x}{\pi^{2} \alpha_{j}}+O_{\varepsilon}\left(x^{3 / 4+\varepsilon}+x^{2 / 3+\varepsilon} \kappa_{j}^{1 / 3}+x^{1 / 2} \kappa_{j}^{1 / 2+\varepsilon}\right), \\
\sum_{n \leq x} c_{j}(n)= & 2 x \operatorname{ch}\left(\pi \kappa_{j}\right) \\
& +O_{\varepsilon}\left(\alpha_{j} \operatorname{ch}\left(\pi \kappa_{j}\right)\left(\kappa_{j}^{1+\varepsilon}+x^{3 / 5+\varepsilon}+x^{1 / 3} \kappa_{j}^{2 / 3+\varepsilon}\right)\right),
\end{aligned}
$$

where $\alpha_{j}$ is defined by $(1.9)$ and $c_{j}(n)$ by (1.20). In the proofs Proskurin used the complex integration method and the bound (1.7) with $\alpha=1 / 4$ for (6.1)-(6.2), and with $\alpha=0$ for (6.3)-(6.4). Since $\alpha=0$ is a form of the Ramanujan-Petersson conjecture for Maass wave forms (Proskurin states, erroneously, that it was proved by N. V. Kuznetsov), it follows that (6.3) and (6.4) are conditional results. Note that the error terms in (6.1) and (6.2) are very large.

The starting point in our proof is, as in [27], the Perron inversion formula for Dirichlet series. For $x^{\varepsilon} \leq T \leq x$ it gives

$$
\sum_{n \leq x} c_{j}(n)=\frac{1}{2 \pi i} \int_{1+\varepsilon-i T}^{1+\varepsilon+i T} C_{j}(s) \frac{x^{s}}{s} d s+O_{\varepsilon}\left(\left|\varrho_{j}(1)\right|^{2} T^{-1} x^{1+2 \alpha+\varepsilon}\right),
$$

where $C_{j}(s)$ is defined by (1.22). It is in the nature of Perron's inversion formula that the exponent $\alpha$ must appear in (6.5). By using the method of Chandrasekharan-Narasimhan [3] this could be avoided, but on the other hand, considerable difficulties would arise since $\kappa_{j}$ is not necessarily fixed. Thus we found it expedient to use (6.5), but unlike Proskurin, who used Perron's formula also to evaluate the sum in (6.1), we find that for this sum the convolution method seems to yield better results. In the course of the proof we shall use (1.23), which was not available to Proskurin, and proceed somewhat differently in treating the integral in (6.5). In fact, it does not seem clear how Proskurin estimates the saddle-point exponential integrals occurring in his proof. 
We apply the residue theorem and the fact that $C_{j}(0)=0$ to obtain

$$
\frac{1}{2 \pi i} \int_{1+\varepsilon-i T}^{1+\varepsilon+i T} C_{j}(s) \frac{x^{s}}{s} d s=2 x \operatorname{ch}\left(\pi \kappa_{j}\right)+I_{1}+I_{2}+I_{3}+I_{4}+I_{5},
$$

where $2 x \operatorname{ch}\left(\pi \kappa_{j}\right)$ comes from the residue of the integrand at $s=1$, and where we set

$$
\begin{gathered}
I_{1}=\frac{1}{2 \pi i} \int_{-\varepsilon-2 i}^{-\varepsilon+2 i} C_{j}(s) \frac{x^{s}}{s} d s \\
I_{2}=\frac{1}{2 \pi i} \int_{-\varepsilon-i T}^{-\varepsilon-2 i} C_{j}(s) \frac{x^{s}}{s} d s, \quad I_{3}=\frac{1}{2 \pi i} \int_{-\varepsilon+2 i}^{-\varepsilon+i T} C_{j}(s) \frac{x^{s}}{s} d s, \\
I_{4}=\frac{1}{2 \pi i} \int_{-\varepsilon+i T}^{1+\varepsilon+i T} C_{j}(s) \frac{x^{s}}{s} d s, \quad I_{5}=\frac{1}{2 \pi i} \int_{1+\varepsilon-i T}^{-\varepsilon-i T} C_{j}(s) \frac{x^{s}}{s} d s .
\end{gathered}
$$

We may write the functional equation (1.24) as

$$
C_{j}(s)=\gamma_{j}(s) C_{j}(1-s),
$$

where

$$
\gamma_{j}(s)=\pi^{4 s-2} \frac{\Gamma\left(\frac{1-s}{2}+i \kappa_{j}\right) \Gamma\left(\frac{1-s}{2}-i \kappa_{j}\right) \Gamma^{2}\left(\frac{1-s}{2}\right)}{\Gamma\left(\frac{s}{2}+i \kappa_{j}\right) \Gamma\left(\frac{s}{2}-i \kappa_{j}\right) \Gamma^{2}\left(\frac{s}{2}\right)} .
$$

Hence, by Stirling's formula, we have

$$
\begin{aligned}
& \gamma_{j}(-\varepsilon+i t) \\
& \quad \ll_{\varepsilon}(|t|+1)^{1+2 \varepsilon}\left(\left|\kappa_{j}-t / 2\right|+1\right)^{1 / 2+\varepsilon}\left(\left|\kappa_{j}+t / 2\right|+1\right)^{1 / 2+\varepsilon} .
\end{aligned}
$$

In view of $(1.23)$ we have $C_{j}(1+\varepsilon+i t) \ll_{\varepsilon} \alpha_{j} \operatorname{ch}\left(\pi \kappa_{j}\right) \kappa_{j}^{\varepsilon}$, hence by (6.6) and (6.7) we have, for $|t| \leq 2$,

$$
C_{j}(-\varepsilon+i t) \ll_{\varepsilon} \alpha_{j} \kappa_{j}^{1+\varepsilon} \operatorname{ch}\left(\pi \kappa_{j}\right),
$$

and the same upper bound holds for $I_{1}$. By applying convexity (the Phragmén-Lindelöf principle) to $C_{j}(s)$ it follows that

$$
\begin{aligned}
I_{4}+I_{5} & \ll T^{-1} \int_{-\varepsilon}^{1+\varepsilon}\left|C_{j}(\sigma+i T)\right| x^{\sigma} d \sigma \\
& \ll_{\varepsilon}\left(\kappa_{j} x\right)^{\varepsilon} \alpha_{j} \operatorname{ch}\left(\pi \kappa_{j}\right)\left(x / T+T+\kappa_{j}\right) .
\end{aligned}
$$

We note that $I_{2}=\bar{I}_{3}$, and use the functional equation (6.6) in $I_{3}$. In view 
of the absolute convergence of the series for $C_{j}(1-s)$ we obtain

$$
I_{3}=\frac{1}{2 \pi x^{\varepsilon}} \sum_{n=1}^{\infty} c_{j}(n) n^{-\varepsilon-1} \int_{2}^{T} \gamma_{j}(-\varepsilon+i t)(x n)^{i t} \frac{d t}{-\varepsilon+i t} .
$$

Stirling's formula gives, for $2 \leq t \leq T$ and $\left|\kappa_{j}-t / 2\right| \geq 2$,

$\gamma_{j}(-\varepsilon+i t)$

$$
=A\left(\kappa_{j}, \varepsilon, t\right) t^{1+2 \varepsilon}\left|\kappa_{j}^{2}-t^{2} / 4\right|^{1 / 2+\varepsilon} e^{i G(t)}\left(1+O\left(\frac{1}{t}\right)+O\left(\frac{1}{\left|\kappa_{j}-t / 2\right|}\right)\right) .
$$

Here

and

$$
A\left(\kappa_{j}, \varepsilon, t\right)=C_{\varepsilon} \exp \left(\frac{i \pi}{4} \operatorname{sgn}\left(\frac{t}{2}-\kappa_{j}\right)\right)
$$

$$
\begin{aligned}
G(t)= & G\left(t, \kappa_{j}\right) \\
:= & 4 t \log \pi-\left(t-2 \kappa_{j}\right) \log \left|t / 2-\kappa_{j}\right|+t-2 \kappa_{j} \\
& -\left(t+2 \kappa_{j}\right) \log \left(t / 2+\kappa_{j}\right)+t+2 \kappa_{j}-2 t \log (t / 2)+2 t .
\end{aligned}
$$

The integral in (6.10) is estimated by the technique similar to the one used for the proof of Theorem 1. The portion for which $\left|\kappa_{j}-t / 2\right|<2$, if it is non-empty, is estimated trivially as $\ll_{\varepsilon} \kappa_{j}^{1 / 2+\varepsilon}$, and its contribution will be $\ll_{\varepsilon} \alpha_{j} \kappa_{j}^{1 / 2+\varepsilon} \operatorname{ch}\left(\pi \kappa_{j}\right)$. The error terms in the formula for $\gamma_{j}(-\varepsilon+i t)$ contribute a total $\ll_{\varepsilon}\left(\kappa_{j} x\right)^{\varepsilon} \alpha_{j} \operatorname{ch}\left(\pi \kappa_{j}\right)\left(\kappa_{j}+T\right)$. The integral coming from the main term is split into $O(\log T)$ subintegrals of the form

$$
\int_{T_{1}}^{T_{2}} \varphi\left(\kappa_{j}, \varepsilon, t\right) e^{i h(t)} d t \quad\left(T_{1}<T_{2} \leq 2 T_{1} \leq T\right),
$$

where $\varphi$ is piecewise monotonic and satisfies

$$
\varphi\left(\kappa_{j}, \varepsilon, t\right) \ll_{\varepsilon} T_{1}^{2 \varepsilon} \max \left(\kappa_{j}, T_{1}\right)^{1+2 \varepsilon} \quad\left(T_{1} \leq t \leq T_{2}\right),
$$

while $h(t):=t \log (x n)+G(t)$ with $G(t)$ given by (6.11). Hence

$$
\begin{gathered}
h^{\prime}(t)=\log \left(\pi^{4} x n\right)-\log \left|t / 2-\kappa_{j}\right|-\log \left(t / 2+\kappa_{j}\right)-2 \log (t / 2), \\
h^{\prime \prime}(t)=\frac{8 \kappa_{j}^{2}-4 t^{2}}{t\left(t^{2}-4 \kappa_{j}^{2}\right)}, \quad h^{\prime \prime \prime}(t)=4 \frac{t^{4}-2 \kappa_{j}^{2} t^{2}+8 \kappa_{j}^{4}}{t^{2}\left(t^{2}-4 \kappa_{j}^{2}\right)^{2}} .
\end{gathered}
$$

Let us fix a small, positive $\delta$. For the values of $t$ for which

$$
\left|h^{\prime \prime}(t)\right| \geq \delta / t
$$

we estimate the corresponding portion of the integral in (6.12) as

$$
\ll_{\varepsilon} T_{1}^{1 / 2+2 \varepsilon} \max \left(\kappa_{j}, T_{1}\right)^{1+2 \varepsilon} \ll_{\varepsilon} T^{1 / 2+2 \varepsilon} \max \left(\kappa_{j}, T\right)^{1+2 \varepsilon} .
$$


The values of $t$ for which (6.13) fails to hold are those which lie in the interval

$$
2\left(\frac{2-\delta}{4-\delta}\right)^{1 / 2} \kappa_{j}<t<2\left(\frac{2+\delta}{4+\delta}\right)^{1 / 2} \kappa_{j}
$$

Hence if $\delta$ is sufficiently small and (6.14) holds, then $h^{\prime \prime \prime}(t) \geq \kappa_{j}^{-2}$. Thus the portion of the integral in $I_{3}$ over the interval in (6.14) (which we denote by $J$ ) can be estimated by the third derivative test (p. 90 of [29]) as

$$
\ll_{\varepsilon} \kappa_{j}^{1+4 \varepsilon} \max _{t \in J}\left|h^{\prime \prime \prime}(t)\right|^{-1 / 3} \ll_{\varepsilon} \kappa_{j}^{5 / 3+4 \varepsilon}
$$

uniformly in $n$. Note that this contribution can exist only if $T \geq \kappa_{j}$. In this case we thus obtain

$$
\begin{aligned}
\sum_{n \leq x} c_{j}(n) & =2 x \operatorname{ch}\left(\pi \kappa_{j}\right) \\
+ & O_{\varepsilon}\left(\alpha_{j} \operatorname{ch}\left(\pi \kappa_{j}\right)\left(\kappa_{j} x\right)^{\varepsilon}\left(x^{\beta} T^{-1}+\kappa_{j}^{5 / 3}+T^{1 / 2} \max \left(\kappa_{j}, T\right)\right)\right),
\end{aligned}
$$

where we have set $\beta=1+2 \alpha$ for brevity. If $T<\kappa_{j}$ then in (6.15) the term $\kappa_{j}^{5 / 3}$ on the right-hand side may be omitted. The optimal $T$ in the range $T \geq \kappa_{j}$ is

$$
T=\max \left(\kappa_{j}, x^{2 \beta / 5}\right)
$$

and we choose this $T$ if $\kappa_{j} \leq x^{3 \beta / 8}$. The optimal $T$ in the range $T<\kappa_{j}$ is

$$
T=\frac{1}{2} \min \left(\kappa_{j}, \kappa_{j}^{-2 / 3} x^{2 \beta / 3}\right),
$$

and we choose this $T$ if $\kappa_{j}>x^{3 \beta / 8}$. In either case $x^{\varepsilon} \leq T \leq x$, as required. Hence, from (6.15), it follows that

$$
\begin{aligned}
& \sum_{n \leq x} c_{j}(n)=2 x \operatorname{ch}\left(\pi \kappa_{j}\right) \\
& \quad+O_{\varepsilon}\left(\alpha_{j} \operatorname{ch}\left(\pi \kappa_{j}\right)\left(\kappa_{j} x\right)^{\varepsilon} \min \left(\kappa_{j}^{5 / 3}+x^{3 \beta / 5}, \kappa_{j}^{-1} x^{\beta}+\kappa_{j}^{2 / 3} x^{\beta / 3}\right)\right) .
\end{aligned}
$$

Finally, we deduce (1.28) from (6.16). By the Möbius inversion formula, (1.20) gives

$$
\left|\varrho_{j}(n)\right|^{2}=\sum_{d^{2} \mid n} \mu(d) c_{j}\left(n / d^{2}\right) .
$$

In view of $\varrho_{j}(n)=\varrho_{j}(1) t_{j}(n)$ and (1.9) it follows that

$$
\Sigma:=\sum_{n \leq x} t_{j}^{2}(n)=\left(\alpha_{j} \operatorname{ch}\left(\pi \kappa_{j}\right)\right)^{-1} \sum_{d^{2} m \leq x} \mu(d) c_{j}(m) .
$$

Suppose now that $\max \left(1, x \kappa_{j}^{-8 /(3 \beta)}\right) \leq y \leq x$. (Values of $y$ in the range $y<x \kappa_{j}^{-8 /(3 \beta)}$ give weaker estimates.) Using (6.16) we obtain 


$$
\begin{aligned}
\Sigma= & \left(\alpha_{j} \operatorname{ch}\left(\pi \kappa_{j}\right)\right)^{-1} \\
& \times\left(\sum_{d \leq \sqrt{y}} \mu(d) \sum_{m \leq x / d^{2}} c_{j}(m)+\sum_{m \leq x / y} c_{j}(m) \sum_{\sqrt{y}<d \leq \sqrt{x / m}} \mu(d)\right) \\
= & \sum_{d \leq \sqrt{y}} \mu(d) \frac{2 x}{\alpha_{j} d^{2}}+O_{\varepsilon}\left(\sum_{d \leq x^{1 / 2} \kappa_{j}^{-4 /(3 \beta)}}\left(\kappa_{j} x\right)^{\varepsilon}\left(\kappa_{j}^{5 / 3}+x^{3 \beta / 5} d^{-6 \beta / 5}\right)\right) \\
& +O_{\varepsilon}\left(\sum_{x^{1 / 2} \kappa_{j}^{-4 /(3 \beta)}<d \leq \sqrt{y}}\left(\kappa_{j} x\right)^{\varepsilon}\left(\kappa_{j}^{-1} x^{\beta} d^{-2 \beta}+\kappa_{j}^{2 / 3} x^{\beta / 3} d^{-2 \beta / 3}\right)\right) \\
& +O_{\varepsilon}\left(\left(\alpha_{j} \operatorname{ch}\left(\pi \kappa_{j}\right)\right)^{-1} \sum_{m \leq x / y} c_{j}(m)(x / m)^{1 / 2}\right) .
\end{aligned}
$$

Here the explicit term and the third error term together give

$$
\frac{12 x}{\pi^{2} \alpha_{j}}+O_{\varepsilon}\left(\kappa_{j}^{\varepsilon} x y^{-1 / 2}\right),
$$

by (1.10) and (1.23). The first and the second error terms together give

$$
O_{\varepsilon}\left(\left(\kappa_{j} x\right)^{\varepsilon}\left(\min \left(x^{1 / 2} \kappa_{j}^{(5 \beta-4) /(3 \beta)}+x^{3 \beta / 5}, \kappa_{j}^{-1} x^{\beta}\right)+\kappa_{j}^{2 / 3} x^{\beta / 3} y^{(3-2 \beta) / 6}\right)\right),
$$

by considering the cases $\kappa_{j} \leq x^{3 \beta / 8}$ and $\kappa_{j}>x^{3 \beta / 8}$ separately. Now the optimal $y$ is $y=x \kappa_{j}^{-2 /(3-\beta)}$ and this satisfies the asumptions on $y$ if $\kappa_{j} \leq$ $x^{(3-\beta) / 2}$. Thus we obtain

$$
\begin{aligned}
\Sigma= & 12 x /\left(\pi^{2} \alpha_{j}\right) \\
& +O_{\varepsilon}\left(x^{\varepsilon}\left(\min \left(x^{1 / 2} \kappa_{j}^{(5 \beta-4) /(3 \beta)}+x^{3 \beta / 5}, \kappa_{j}^{-1} x^{\beta}\right)+x^{1 / 2} \kappa_{j}^{1 /(3-\beta)}\right)\right) .
\end{aligned}
$$

Then (1.28) follows, since $\beta=1+2 \alpha$.

The asymptotic formula (1.28) with $\alpha=1 / 4(\alpha=5 / 28$ is known by [2]) improves (6.1), while (1.28) with $\alpha=0$ improves (6.3).

In conclusion, it may be remarked that in the course of the proof we used the trivial bound $\sum_{n<x} \mu(n) \ll x$. However, if we assume the Riemann hypothesis, which is equivalent to the statement (see Ch. 14 of [29]) that

$$
\sum_{n \leq x} \mu(n) \ll_{\varepsilon} x^{1 / 2+\varepsilon}
$$

then a conditional improvement of Theorem 3 may be obtained.

\section{References}

[1] D. Bump, The Rankin-Selberg method: a survey, in: Number Theory, Trace Formulas and Discrete Groups, Sympos. in honor of A. Selberg (Oslo, 1987), Academic Press, Boston, 1989, 49-109. 
[2] D. Bump, W. Duke, D. Ginsburg, J. Hoffstein and H. Iwaniec, An estimate for Fourier coefficients of Maass wave forms, Duke Math. J. 66 (1992), 75-81.

[3] K. Chandrasekharan and R. Narasimhan, Functional equations with multiple gamma-factors and the average order of arithmetical functions, Ann. of Math. 76 (1962), 93-136.

[4] - - - On the mean value of the error term for a class of arithmetical functions, Acta Math. 112 (1964), 41-67.

[5] J.-M. Deshouillers and H. Iwaniec, The non-vanishing of the Rankin-Selberg zeta-functions at special points, in: Selberg Trace Formulas, Contemp. Math. 53, Amer. Math. Soc., Providence, R.I., 1986, 51-95.

[6] A. Erdélyi et al., Higher Transcendental Functions, Vol. 2, McGraw-Hill, New York, 1953.

[7] J. L. Hafner and A. Ivić, On sums of Fourier coefficients of cusp forms, Enseign. Math. 35 (1989), 375-382.

[8] A. Ivić, The Riemann Zeta-Function, Wiley, New York, 1985.

[9] - Lectures on Mean Values of the Riemann Zeta-Function, Tata Inst. Fund. Res. Lect. Notes 82, Bombay, 1991, Springer, Berlin, 1991.

[10] A. Ivić and Y. Motohashi, A note on the mean value of the zeta and L-functions. VII, Proc. Japan Acad. Ser. A 66 (1990), 150-152.

[11] H. Iwaniec, Fourier coefficients of cusp forms and the Riemann zeta-function, Séminaire de Théorie des Nombres, Université Bordeaux 1979/80, exp. no. 18.

[12] - Non-holomorphic modular forms and their applications, in: Modular Forms, R. A. Rankin (ed.), Sympos. Durham 1983, Halsted Press, New York, 1984, 157196.

[13] - Promenade along modular forms and analytic number theory, in: Topics in Analytic Number Theory, S. W. Graham and J. D. Vaaler (eds.), University of Texas Press, Austin, Texas, 1985, 221-303.

[14] - Small eigenvalues of the Laplacian for $\Gamma_{0}(N)$, Acta Arith. 56 (1990), 65-82.

[15] - , The spectral growth of automorphic L-functions, J. Reine Angew. Math. 428 (1992), 139-159.

[16] M. Jutila, A Method in the Theory of Exponential Sums, Tata Inst. Fund. Res. Lect. Notes 80, Bombay, 1987, Springer, Berlin, 1987.

[17] N. V. Kuznetsov, Petersson hypothesis for forms of weight zero and Linnik's conjecture. Sums of Kloosterman sums, Math. USSR-Sb. 39 (1981), 299-342.

[18] - Mean value of Hecke series associated with cusp forms of weight zero, Zap. Nauchn. Sem. LOMI 109 (1981), 93-130 (in Russian).

[19] - Convolution of the Fourier coefficients of the Eisenstein-Maass series, ibid. 129 (1983), 43-84 (in Russian).

[20] T. Meurman, On exponential sums involving the Fourier coefficients of Maass wave forms, J. Reine Angew. Math. 384 (1985), 192-207.

[21] - On the mean square of the Riemann zeta-function, Quart. J. Math. (Oxford) (2) 38 (1987), 337-343.

[22] C. Moreno and F. Shahidi, The L-functions $L\left(s, \operatorname{Sym}^{m}(r), \pi\right)$, Canad. Math. Bull. 28 (1985), 405-410.

[23] Y. Motohashi, A note on the mean value of the zeta and L-functions. VI, Proc. Japan Acad. Ser. A 65 (1989), 273-275.

[24] - An explicit formula for the fourth power mean of the Riemann zeta-function, Acta Math. 170 (1993), 181-220.

[25] M. R. Murty, On the estimation of eigenvalues of Hecke operators, Rocky Mountain J. Math. 15 (1985), 521-533. 
[26] E. Preissmann, Sur la moyenne quadratique du terme de reste du problème du cercle, C. R. Acad. Sci. Paris Sér. I 306 (1988), 151-154.

[27] N. V. Proskurin, Convolution of Dirichlet series with Fourier coefficients of parabolic forms of weight zero, Zap. Nauchn. Sem. LOMI 93 (1980), 204-217 (in Russian).

[28] R. A. Rankin, Contributions to the theory of Ramanujan's function $\tau(n)$ and similar arithmetical functions II. The order of the Fourier coefficients of integral modular forms, Proc. Cambridge Philos. Soc. Math. 35 (1939), 357-372.

[29] E. C. Titchmarsh, The Theory of the Riemann Zeta-Function, 2nd ed., Clarendon Press, Oxford, 1986.

[30] K.-C. Tong, On divisors problems III, Acta Math. Sinica 6 (1956), 515-541 (in Chinese).

[31] G. N. Watson, A Treatise on the Theory of Bessel Functions, 2nd ed., Cambridge University Press, Cambridge, 1944.

KATEDRA MATEMATIKE RGF-A UNIVERSITETA U BEOGRADU DJUŠINA 7, 11000 BEOGRAD (SERBIA) YUGOSLAVIA

\section{DEPARTMENT OF MATHEMATICS UNIVERSITY OF TURKU SF-20500 TURKU} FINLAND 